\title{
Alcohol intake and gastric cancer: Meta-analyses of published data versus individual participant data pooled analyses (StoP Project)
}

Ana Ferro ${ }^{\mathrm{a}}$, Samantha Morais ${ }^{\mathrm{a}}$, Matteo Rota ${ }^{\mathrm{c}, \mathrm{d}}$, Claudio Pelucchi ${ }^{\mathrm{d}}$, Paola Bertuccio ${ }^{\mathrm{d}}$, Rossella Bonzi ${ }^{\mathrm{d}}$, Carlotta Galeone ${ }^{\mathrm{d}}$, Zuo-Feng Zhang ${ }^{\mathrm{e}}$, Keitaro Matsuo ${ }^{\mathrm{f}}$, Hidemi Ito $^{\mathrm{f}}$, Jinfu $\mathrm{Hu}^{\mathrm{g}}$, Kenneth C. Johnson ${ }^{\mathrm{h}}$, Guo-Pei Yu ${ }^{\mathrm{i}}$, Domenico Palli ${ }^{\mathrm{j}}$, Monica Ferraroni ${ }^{\mathrm{d}}$, Joshua Muscat ${ }^{\mathrm{k}}$, Reza Malekzadeh ${ }^{1}$, Weimin $\mathrm{Ye}^{\mathrm{m}}$, Huan Song $^{\mathrm{m}, \mathrm{n}}$, David Zaridze ${ }^{\mathrm{o}}$, Dmitry Maximovitch $^{\circ}$, Nerea Fernández de Larrea ${ }^{\mathrm{p}, \mathrm{q}}$, Manolis Kogevinas ${ }^{\mathrm{q}, \mathrm{r}, \mathrm{s}, \mathrm{t}}$, Jesus Vioque ${ }^{\mathrm{u}}$, Eva M. Navarrete-Muñoz ${ }^{\mathrm{u}}$, Mohammadreza Pakseresht ${ }^{1, v, w}$, Farhad Pourfarzi ${ }^{1, \mathrm{x}}$, Alicja Wolk ${ }^{\mathrm{y}, \mathrm{H}}$, Nicola Orsini ${ }^{\mathrm{y}}$, Andrea Bellavia $^{\mathrm{y}}$, Niclas Håkansson ${ }^{\mathrm{y}}$, Lina $\mathrm{Mu}^{\mathrm{z}}$, Roberta Pastorino ${ }^{\mathrm{A}}$, Robert C. Kurtz ${ }^{\mathrm{B}}$, Mohammad H. Derakhshan $^{\mathrm{C}, 1}$, Areti Lagiou ${ }^{\mathrm{D}}$, Pagona Lagiou ${ }^{\mathrm{E}, \mathrm{F}}$, Paolo Boffetta ${ }^{\mathrm{G}}$, Stefania Boccia ${ }^{\mathrm{A}}$, Eva Negri ${ }^{\mathrm{c}}$, Carlo La Vecchia ${ }^{\mathrm{d}}$, Bárbara Peleteiro ${ }^{\mathrm{a}, \mathrm{b}}$, Nuno Lunet ${ }^{\mathrm{a}, \mathrm{b}, *}$

a EPIUnit - Instituto de Saúde Pública, Universidade do Porto, Rua das Taipas, $n^{\circ} 135$, 4050-600, Porto, Portugal

b Departamento de Ciências da Saúde Pública e Forenses e Educação Médica, Faculdade de Medicina, Universidade do Porto, Al. Prof. Hernâni Monteiro, 4200-319, Porto, Portugal

${ }^{\mathrm{c}}$ Department of Biomedical and Clinical Sciences, University of Milan, Milan, Italy

' Department of Clinical Sciences and Community Health (DISCCO), University of Milan, Milan, Italy

e Department of Epidemiology, UCLA Fielding School of Public Health and Jonsson Comprehensive Cancer Center, Los Angeles, CA, USA

${ }^{\mathrm{f}}$ Division of Molecular Medicine, Aichi Cancer Center Research Institute, Nagoya, Japan

${ }^{\mathrm{g}}$ Department of Epidemiology, Harbin Medical University, Harbin, China

${ }^{\text {h }}$ School of Epidemiology, Public Health and Preventive Medicine, Faculty of Medicine, University of Ottawa, Ottawa, Ontario, Canada

${ }^{\mathrm{i}}$ Medical Informatics Center, Peking University, Peking, China

${ }^{\mathrm{j}}$ Molecular and Nutritional Epidemiology Unit, Cancer Research and Prevention Institute - Istituto per lo Studio e la Prevenzione Oncologica (ISPO), Florence, Italy

${ }^{\mathrm{k}}$ Department of Public Health Sciences, The Pennsylvania State University College of Medicine, Penn State Hershey Medical Center, Hershey, PA, USA

${ }^{1}$ Digestive Oncology Research Center, Digestive Disease Research Institute, Tehran University of Medical Sciences, Tehran, Iran

${ }^{m}$ Department of Medical Epidemiology and Biostatistics, Karolinska Institutet, Stockholm, Sweden

${ }^{n}$ Center of Public Health Sciences, Faculty of Medicine, University of Iceland, Reykjavík, Iceland

${ }^{\circ}$ Department of Epidemiology and Prevention, Russian N.N. Blokhin Cancer Research Center, Moscow, Russia

P Environmental and Cancer Epidemiology Unit, National Center of Epidemiology, Instituto de Salud Carlos III, Madrid, Spain

${ }^{\mathrm{q}}$ CIBER Epidemiología y Salud Pública (CIBERESP), Madrid, Spain

${ }^{\mathrm{r}}$ ISGlobal, Centre for Research in Environmental Epidemiology (CREAL), Barcelona, Spain

s IMIM (Hospital del Mar Medical Research Institute), Barcelona, Spain

${ }^{\mathrm{t}}$ Universitat Pompeu Fabra (UPF), Barcelona, Spain

' Miguel Hernandez University and ISABIAL-FISABIO Foundation, Campus San Juan, Alicante, Spain

${ }^{\mathrm{v}}$ Department of Agricultural, Food and Nutritional Sciences, University of Alberta, Edmonton, Alberta, Canada

${ }^{\mathrm{w}}$ Nutritional Epidemiology Group, Centre for Epidemiology and Biostatistics, University of Leeds, Leeds, UK

${ }^{\mathrm{x}}$ Digestive Diseases Research Center, Ardabil University of Medical Sciences, Ardabil, Iran

${ }^{\mathrm{y}}$ Unit of Nutritional Epidemiology, Institute of Environmental Medicine, Karolinska Institutet, Stockholm, Sweden

${ }^{\mathrm{z}}$ Department of Epidemiology and Environmental Health, School of Public Health and Health Professions, University at Buffalo, Buffalo, NY, USA

${ }^{A}$ Section of Hygiene - Institute of Public Health; Università Cattolica del Sacro Cuore, IRCCS Fondazione Policlinico "Agostino Gemelli”, L.go F. Vito, 1 - 00168, Rome, Italy

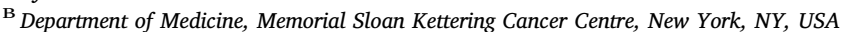

${ }^{\mathrm{C}}$ Institute of Cardiovascular \& Medical Sciences, University of Glasgow, Glasgow, United Kingdom

D Department of Public Health and Community Health, School of Health Professions, Athens Technological Educational Institute, Athens, Greece

${ }^{\mathrm{E}}$ Department of Hygiene, Epidemiology and Medical Statistics, School of Medicine, National and Kapodistrian University of Athens, Athens, Greece

${ }^{\mathrm{F}}$ Department of Epidemiology, Harvard T.H. Chan School of Public Health, Boston, MA, USA

${ }^{\mathrm{G}}$ The Tisch Cancer Institute, Icahn School of Medicine at Mount Sinai, New York, NY, USA

${ }^{\mathrm{H}}$ Department of Surgical Sciences, Uppsala University, Uppsala Sweden

\footnotetext{
* Corresponding author at: Departamento de Ciências da Saúde Pública e Forenses e Educação Médica, Faculdade de Medicina, Universidade do Porto, Alameda Prof. Hernâni Monteiro, 4200-319, Porto, Portugal.

E-mail address: nlunet@med.up.pt (N. Lunet).
} 


\section{A R T I C L E I N F O}

\section{Keywords:}

Gastric cancer

Individual participant data

Meta-analysis

Pooled analysis

Alcohol

Publication bias

\begin{abstract}
A B S T R A C T
Background: Individual participant data pooled analyses allow access to non-published data and statistical reanalyses based on more homogeneous criteria than meta-analyses based on systematic reviews. We quantified the impact of publication-related biases and heterogeneity in data analysis and presentation in summary estimates of the association between alcohol drinking and gastric cancer.

Methods: We compared estimates obtained from conventional meta-analyses, using only data available in published reports from studies that take part in the Stomach Cancer Pooling (StoP) Project, with individual participant data pooled analyses including the same studies.

Results: A total of 22 studies from the StoP Project assessed the relation between alcohol intake and gastric cancer, 19 had specific data for levels of consumption and 18 according to cancer location; published reports addressing these associations were available from 18, 5 and 5 studies, respectively. The summary odds ratios [OR, (95\%CI)] estimate obtained with published data for drinkers vs. non-drinkers was $10 \%$ higher than the one obtained with individual StoP data [18 vs. 22 studies: $1.21(1.07-1.36)$ vs. $1.10(0.99-1.23)]$ and more heterogeneous ( $\mathrm{I}^{2}: 63.6 \%$ vs $\left.54.4 \%\right)$. In general, published data yielded less precise summary estimates (standard errors up to 2.6 times higher). Funnel plot analysis suggested publication bias.

Conclusion: Meta-analyses of the association between alcohol drinking and gastric cancer tended to overestimate the magnitude of the effects, possibly due to publication bias. Additionally, individual participant data pooled analyses yielded more precise estimates for different levels of exposure or cancer subtypes.
\end{abstract}

\section{Introduction}

Systematic reviews have the potential to settle controversies arising from apparently conflicting findings and to answer questions not directly addressed by single studies, as well as to enhance the precision of effect measures [1-4]. Individual participant data pooled analyses are considered more capable of overcoming some of the limitations of systematic reviews and meta-analyses of published data [5], since they allow access to data not previously published and statistical reanalysis based on more homogeneous criteria [3]. However, individual participant data pooled analyses require much more complex and costly management of data, as well as coordination of the underlying consortium of research groups, and the gains in terms of precision and validity of the results may be expected to vary with the topic being addressed. Comparisons of individual participant data pooled analyses with meta-analyses based on the published data from the same studies contribute to understand the extent to which conventional meta-analyses may be biased or lack statistical power, and different results may be expected for distinct research questions.

The World Cancer Research Fund reported evidence of a probable association between alcohol drinking and gastric cancer in April 2016. There were no individual participant pooled analyses for this exposure in that update [6]. The Stomach Cancer Pooling (StoP) Project [7] has recently published a pooled analysis assessing the association between alcohol intake and gastric cancer, based on information from more than 10,000 cases and 26,000 controls evaluated in 20 studies conducted in 10 countries. Heavy drinkers, defined as those drinking more than six drinks per day, had a significant excess risk of gastric cancer of approximately $50 \%$, compared to never drinkers [8]. That study includes results from studies that never addressed this topic before and was based on more homogeneous methodological approaches, namely regarding the definition of alcohol intake and control of confounding. Therefore, it adds to previous evidence supporting a potential role of alcohol as a probable risk factor for gastric cancer [9-14], specifically for three or more drinks per day [6].

In the present study, we provide quantitative estimates of the impact of publication biases and heterogeneity in data analysis and presentation, in the summary estimates of the association between alcohol drinking and gastric cancer obtained from conventional meta-analyses. We used data available in previously published reports from studies that take part in the Stomach Cancer Pooling (StoP) Project, for comparison with individual participant data pooled analyses including the same studies.

\section{Methods}

\subsection{Individual participant data meta-analysis}

The StoP Project is a consortium of case-control studies (including nested case-control within cohort studies), including at least 80 incident, histologically confirmed, gastric cancer cases [7]. The StoP Project received ethical approval from the University of Milan Review Board.

The first release of the StoP Project dataset included 23 case-control studies, comprising 10,290 cases (6,804 men, 3,486 women) and 26,145 controls $(15,600$ men, 10,545 women) from Greece [15], Italy (four studies) [16-19], Portugal [20], Russia [21], Spain (two studies) $[22,23]$, Sweden (three studies, two of which were nested in cohort studies) [24,25], China (four studies) [26-29], Iran (three studies) [30-32], Japan [33], Canada [34] and the United States of America (USA) (two studies, one of them unpublished) [35].

The association between alcohol drinking and gastric cancer was estimated through a two-stage modeling approach [8]. Briefly, in the first stage, the association between alcohol drinking and gastric cancer for each study was assessed through multivariable logistic regression models that included, whenever available, terms for age, sex, education/social class, smoking, fruit and vegetable consumption, study center (for multicenter studies), as well as terms for the matching variables, when applicable. In the second stage, the pooled effects estimates were computed using a random-effect models, through the DerSimonian and Laird method [36]. This was performed for the comparison of the following levels of exposure: 1) drinkers vs. nondrinkers; 2) drinkers of less than one drink per day vs. non-drinkers; 3) drinkers of one to four drinks per day vs. non-drinkers; 4) drinkers of over four drinks per day vs. non-drinkers. Heterogeneity was quantified using the $\mathrm{I}^{2}$ statistic [37].

\subsection{Meta-analysis of published data}

\subsubsection{Search strategy}

The strategy to identify all published reports from the 23 studies included in the first version of the StoP Project database is depicted in Supplementary Fig. 1.

We searched PubMed, from inception to December 31, 2016, and conducted forward citation tracking of the reference provided in the StoP Project presentation paper to identify papers based on the same dataset, through Google Scholar and Web of Science ${ }^{\mathrm{TM}}$. The responsible investigators for each study were then asked to confirm if all published reports of results from their study had been included, and no additional 
articles were identified.

\subsubsection{Data extraction and meta-analysis}

Two investigators (AF, SM) evaluated independently the selected studies to extract the following data from the original reports: first author, publication year, country, geographic area, number of cases and controls, period of data collection, definition of alcohol consumption, stratification variables and relative risk estimates (odds and hazard ratios) for the association between alcohol drinking and gastric cancer, along with the corresponding confidence intervals. Preference was given to estimates adjusted for the largest number of confounders, although crude estimates or data to compute them could also be extracted when only these were available.

The assessment of alcohol consumption was mainly done through questionnaires, with cases and controls describing frequency and total amount of alcohol intake. However, the description of alcohol drinking habits varied substantially among the reports. When possible, we chose non-drinkers as the reference category, described in the original reports as "non-drinkers", "alcohol drinking: no", "alcohol drinking: never". In several studies, occasional drinkers or drinkers of small quantities of alcohol (defined according to quintiles of consumption) were included in the reference category. In the case of the study Italy 3 [17], nondrinkers included "individuals whose alcohol intake was less than seven grams per day", and in the study Spain 2 [23] never drinkers were those who consumed less than one drink per month; the studies China 1 [26] and 4 [29], Greece [15] and Italy 2 [19] had a category of low consumption, either defined by the lowest quintile or the lowest amount of alcohol intake, as reference.

Different units were used to express alcohol drinking: grams per day, grams per week, kg per year, times per day, times per week, drinks per day, servings per week, glasses per week and ml per month. We converted them to drinks per day assuming the following equivalences: 1 drink $=12 \mathrm{~g}$ of pure ethanol and $1 \mathrm{ml}=0.8 \mathrm{~g}$ of ethanol [38]. In order to identify categories of current alcohol consumption corresponding to the exposure closest to less than one drink per day, one to four drinks per day and more than four drinks per day, we assumed that each category corresponded to an exposure equal to the midpoint of the respective category range and that the open-ended categories had the amplitude of the preceding stratum (e.g.: for surveys reporting $\leq 2$, $2-4,4-6, \geq 6$ drinks per day, 1 and 7 were the midpoints assigned to the lowest and highest category, respectively).

Data were also extracted according to cancer location within the stomach. For the purpose of analyses, results referring to "cardia" or "esophagus and gastric cardia" cancers were taken as equivalent to cancer of the gastric cardia, and "distal", "non-cardia" or "all others" as equivalent to cancers not located in the cardia.

Meta-analyses were conducted following as close as possible the analyses described for the individual participant data pooled analyses. The DerSimonian and Laird method [36] was used to pool the estimates calculated for each study. Heterogeneity was quantified using the $\mathrm{I}^{2}$ statistic [37].

\subsection{Comparison between meta-analyses of published data and of individual participant data}

The meta-analyses of published data and individual participant data were compared regarding the number of studies included, the estimates obtained and corresponding precision, as well as heterogeneity of results. For each of these items, the ratios of the values obtained in conventional meta-analysis and individual participant data pooled analyses (ratio MA/StoP) were computed, assuming the latter as the gold standard.
StoP Project Estimates

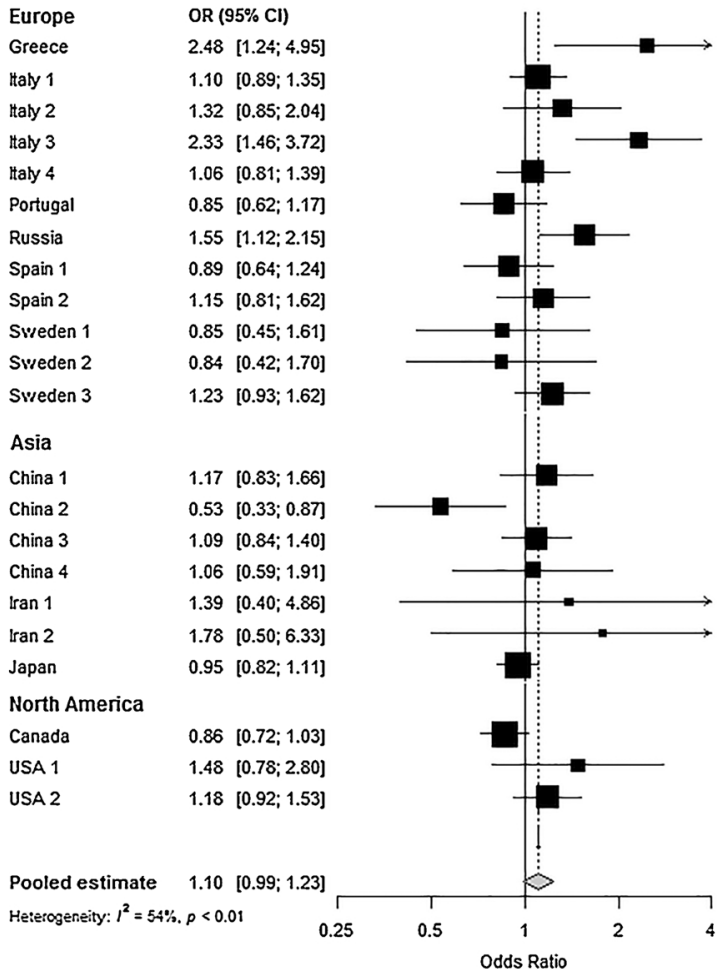

Conventional meta-analysis

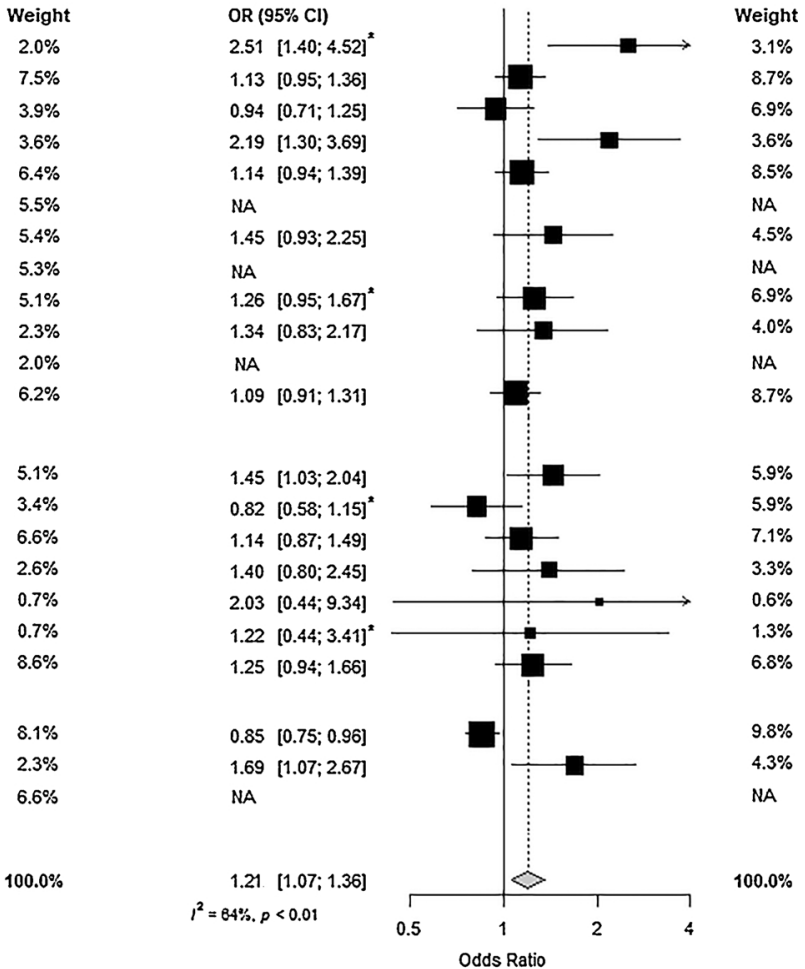

Fig. 1. Forest plots describing the association between alcohol drinking and gastric cancer, with non drinkers as reference, using the estimates from the Stomach Cancer Pooling (StoP) Project database and from the published reports of the same studies. 


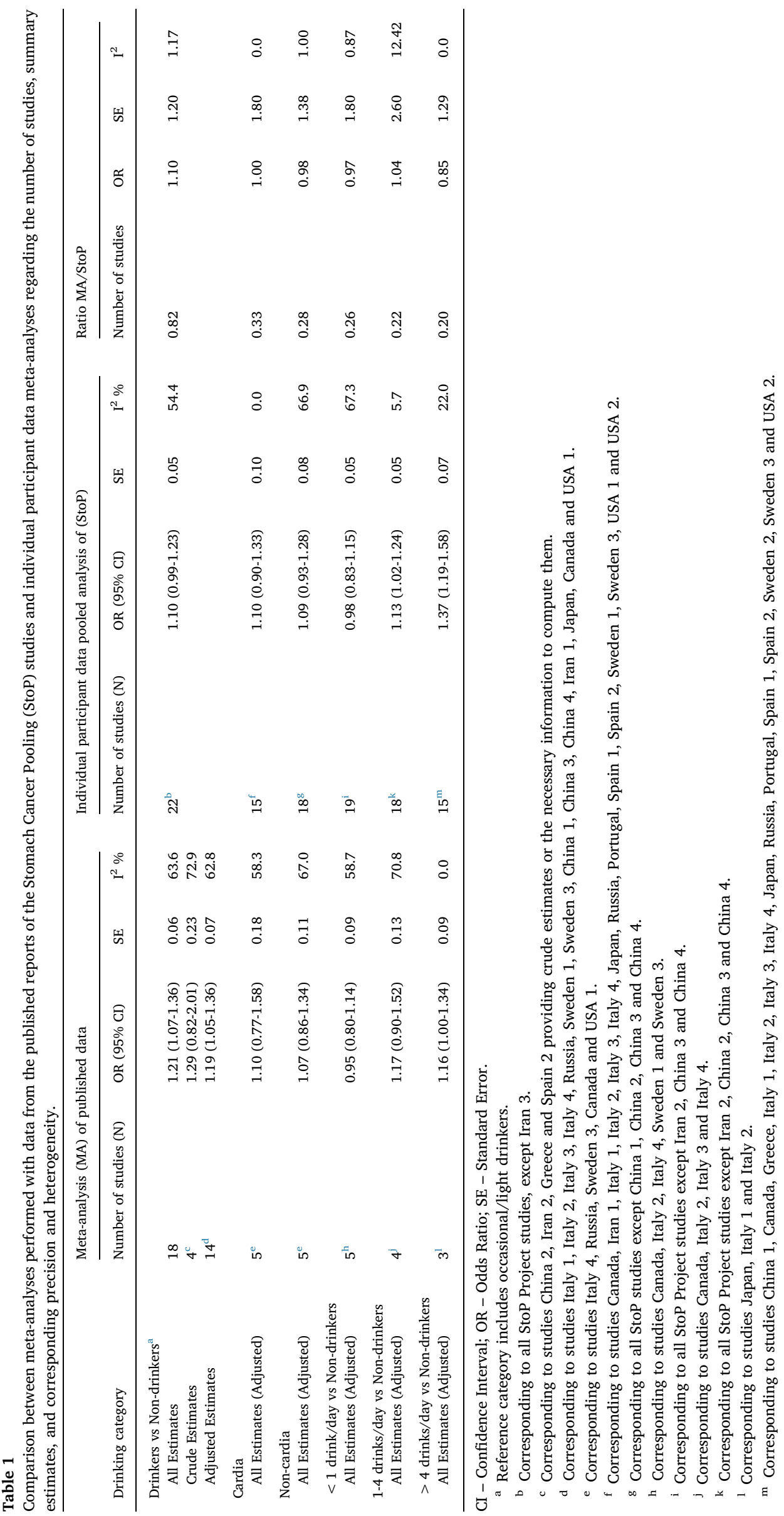


Funnel plots and Egger's regression asymmetry test were used for the assessment of publication bias [4].

All statistical analyses were performed using STATA $^{\circledR}$ statistical software package version 11.2 (StataCorp., College Station, Texas, USA).

\section{Results}

\subsection{Individual-participant data pooled analysis}

Twenty-two out of the 23 studies included in the first version of the StoP Project database had information to compute an estimate of the risk of gastric cancer for drinkers vs. non-drinkers [Odds Ratio (OR) (95\% Confidence Interval (CI)): $1.10(0.99-1.23)]$ (Fig. 1 and Supplementary Table 1).

An excess of gastric cancer risk was observed in drinkers compared to non-drinkers (Table 1), with the strongest association being observed for drinkers of more than four drinks per day [1.37 (1.19-1.58)].

\subsection{Meta-analysis of published data}

A total of 192 reports from the set of 23 studies included in the first release of the StoP Project dataset were identified in the systematic literature search: two from Greece, 86 from Italy, eight from Portugal,
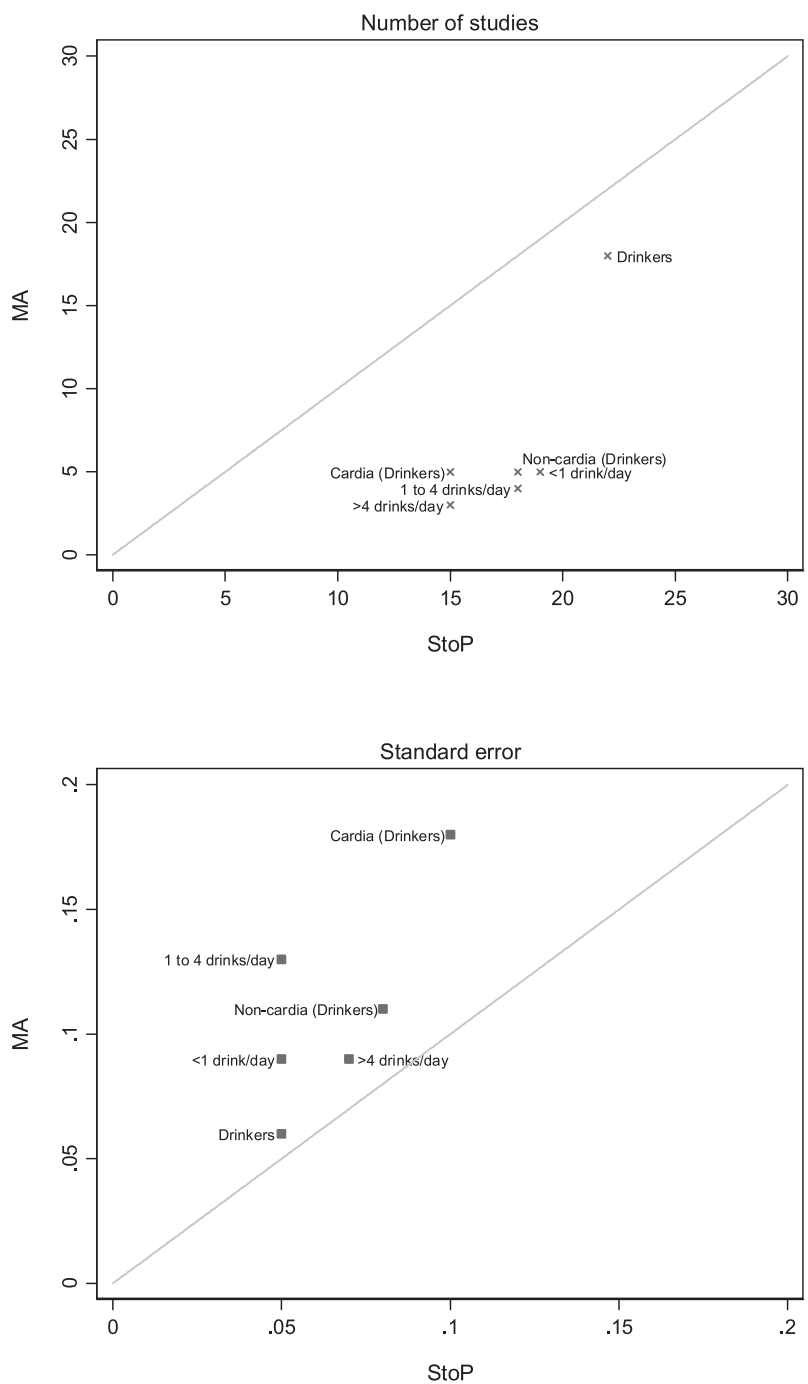

four from Russia, four from Spain, 29 from Sweden, 22 from China, three from Iran, 18 from Japan, 10 from Canada and six from the USA (Supplementary Table 2).

The analyses were performed using information extracted from 21 reports, providing data for 18 of the 23 original studies from StoP. Five studies (Iran 3, Portugal, Spain 1, Sweden 2 and USA 2) had no published data on the relation between alcohol drinking and gastric cancer. The reports from four studies (Greece, Spain 2, China 2 and Iran 2) provided only crude estimates of the association between alcohol drinking and gastric cancer, or the necessary information to compute them. A detailed description of each study and corresponding results included in the conventional meta-analysis is provided in Supplementary Table 2. The corresponding summary OR estimates for the comparison of drinkers vs. non-drinkers are presented in Supplementary Table 1 and depicted in Fig. 1; study specific estimates obtained from published reports more often supported a stronger association between alcohol drinking and gastric cancer.

\subsection{Comparison between conventional meta-analyses and individual participant data pooled analyses}

Table 1 and Fig. 2 show the comparison between conventional meta-analyses and individual participant data pooled analyses.

Data on the comparison between drinkers and non-drinkers were
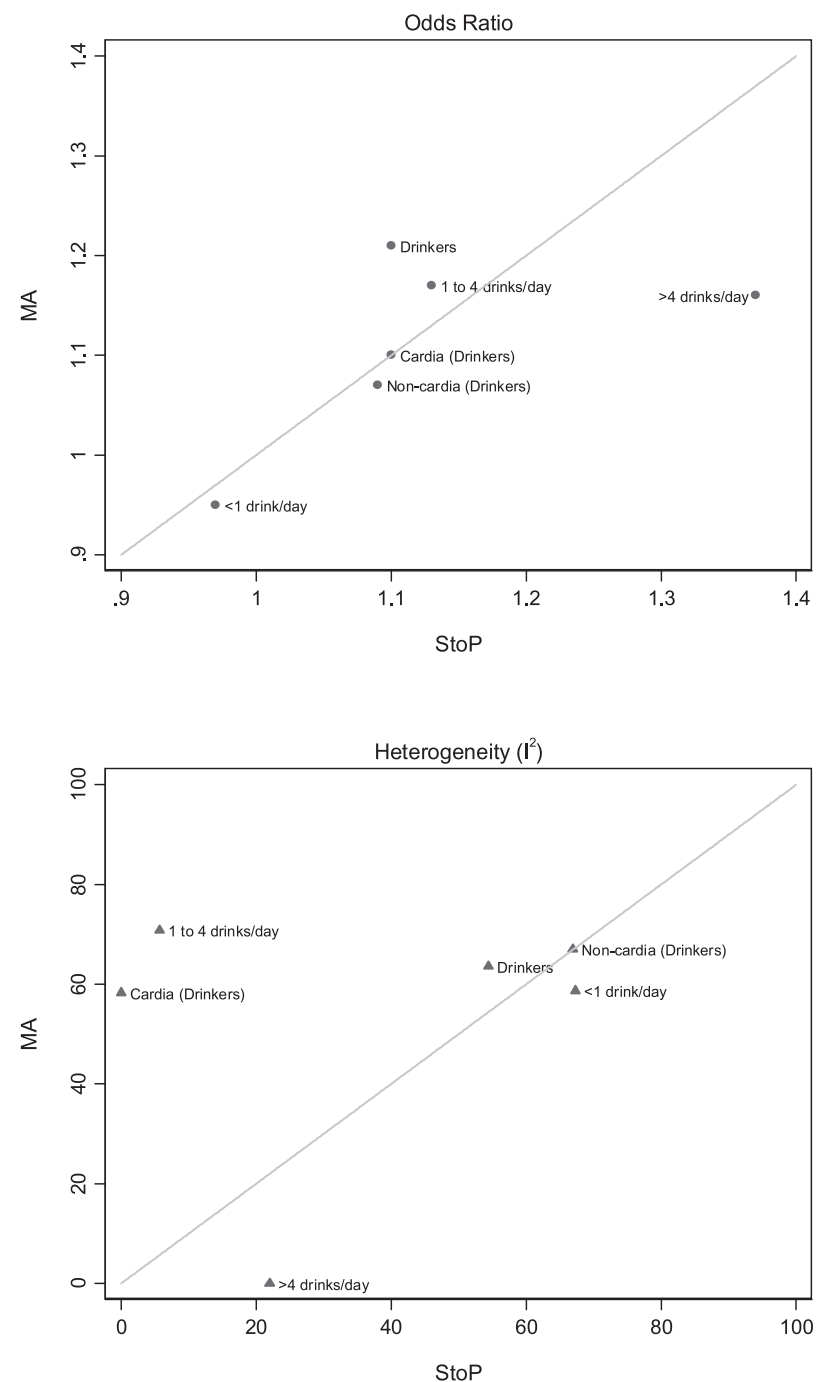

Fig. 2. Graphic representation of the results of conventional meta-analyses (MA) and individual participant data pooled analyses (StoP) for the number of studies and odds ratio, standard error and heterogeneity estimates, according to the different levels of exposure compared with non-drinkers. 
available for a larger number of studies (18 of 22). The summary OR obtained with published data was about $10 \%$ higher than the one obtained with the StoP data (1.21 vs. 1.10$)$ with a higher standard error (ratio $\mathrm{MA} / \mathrm{StoP}=1.20$ ) and greater heterogeneity (ratio MA/ StoP $=1.17$ ). Sensitivity analyses conducted by removing each study at a time did not meaningfully change the results obtained with the StoP data or the meta-analyses of published data. Among the published reports there were 14 studies providing adjusted estimates; the corresponding summary estimates were also higher than those obtained with the StoP data from the same studies (1.19 vs 1.14 ).

For specific cancer locations and levels of exposure, the differences between meta-analyses were mainly regarding the number of studies and the precision of the estimates. For gastric cardia and non-cardia cancers, the ratios MA/StoP were 0.33 and 0.28 , for the number of studies, respectively, and the standard error was $80 \%$ and $38 \%$ higher, respectively, than the ones from the individual participant data pooled analyses. Regarding the number of drinks per day, only around one-fifth of the StoP studies had published reports with this information, resulting in more imprecise estimates than the ones from the individual participant pooled analyses. For example, only four out of 18 studies had published data for drinkers of one to four drinks per day.

The visual inspection of the funnel plot and Egger's test $(P=0.001)$ are suggestive of publication bias only for the meta-analysis of published data (Fig. 3).

\section{Discussion}

We observed that, for the comparison between drinkers and nondrinkers, the use of published data overestimated the association with gastric cancer. For various categories of exposure considered, the estimates obtained with both published and individual participant data were similar, although the latter were generally less heterogeneous and more precise.

Most previously published meta-analyses addressing the relation between heavy alcohol drinking and gastric cancer reported significant positive associations, particularly with an increasing number of drinks per day [9-11,13,14], although with lower magnitude than the estimates obtained in the present individual participant pooled analyses. Tramacere et al [9], using information from 10 case-control studies, estimated a 1.22 (95\% confidence interval: 0.98-1.52) OR for heavy drinkers (defined as those drinking more than four drinks per day) and Bagnardi et al [11], with 11 case-control studies, estimated a similar estimate [Relative risk (RR) (95\% CI): 1.22 (0.97-1.54)] for the same amount of drinks. Meta-analyses that assessed the association using information from cohort studies obtained summary estimates lower than the ones from the present analyses, $[9,11,12]$ though in the most recent meta-analysis a statistically significant association was observed only among cohort studies, for categories of heavy drinking [11].

The differences between the summary estimates obtained in the previous meta-analyses and in the present pooled analysis may be explained by differences in the characteristics of the original studies included and by avoidance of publication bias and improved homogeneity. However, comparisons between published and unpublished data reflect essentially publication biases and heterogeneity in data analysis and presentation, and therefore the differences may vary across different sets of studies, according to the extent to which less favorable results are not published or made available with less detail.

For the present study, we obtained the most complete data possible from all the eligible studies of the StoP Project and we were able to set the results based on a reanalysis of the original studies, using uniform criteria, as the reference for comparison with a conventional metaanalysis. Our results could overestimate the differences between the two strategies of synthesis, because authors of a conventional metaanalysis may contact the authors of the original studies seeking additional data to complement those available in the published reports. However, a large proportion of systematic reviews are based only on published data, and attempts to retrieve additional data by contacting the authors from the original studies are often unsuccessful $[39,40]$. When comparisons between meta-analysis and pooled analysis are based on a different number of studies, disagreements may also reflect differences in the characteristics of the studies considered for each of these strategies of synthesis. In the present study, the OR estimates were very similar for the meta-analysis and the pooled analysis, and the most important differences were in terms of enhanced precision and homogeneity among the pooled analysis.

The definition of non-drinkers varied substantially across studies; in some cases they included infrequent drinkers [15,19,23,41,42], while in others the term non-drinker was not further defined $[43,44]$. As it

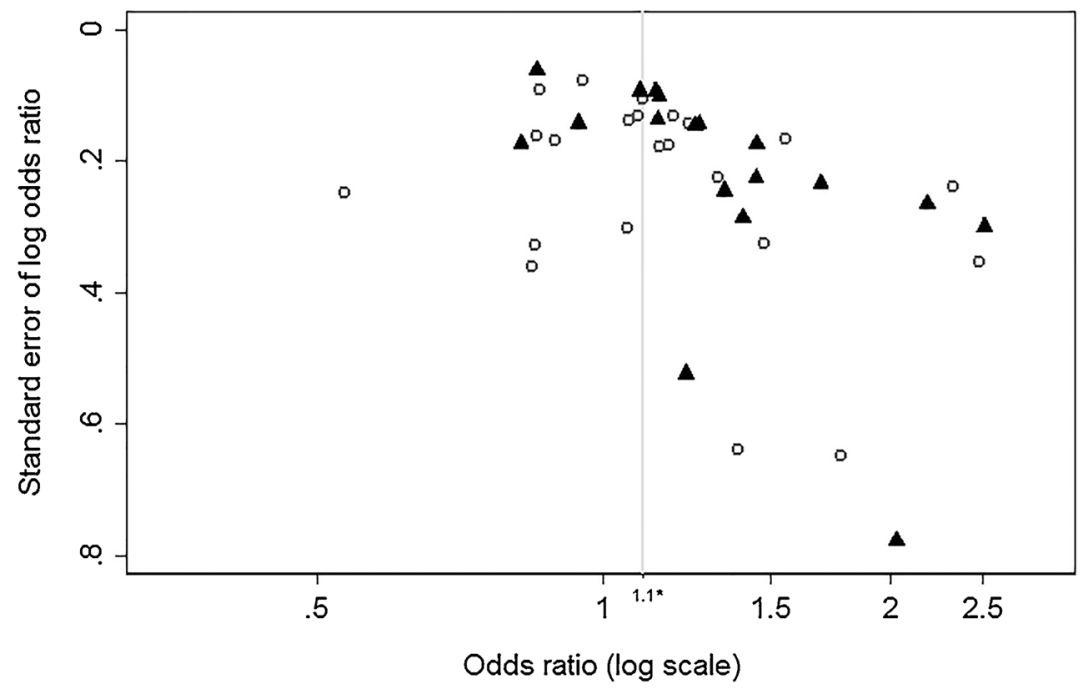

- Meta-analysis of published data o StoP

* Summary odds ratio estimate obtained from the pooled analysis of individual participant data (StoP)

Egger's regression asymmetry test: Meta-analysis of published data $(p=0.001) ; \operatorname{StoP}(p=0.106)$

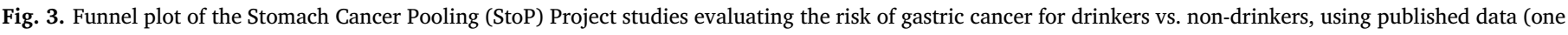
estimate per study) and individual participant data from the StoP Project database. 
was not possible to organize, from the published data, all individuals into a common definition of non-drinker, the specific definition from each study was used, which may have contributed to the observed heterogeneity. This is a common issue when analyzing the effect of alcohol intake and is discussed in several meta-analyses for the association between alcohol and cancer $[14,45,46]$. Such a lack of a common definition should have led to an underestimation rather than an overestimation of the association with alcohol in the meta-analyses as compared to the pooled analyses. In addition, the latter are expected to yield more precise and valid RR estimates, namely by accounting for the potential confounding effect of socioeconomic status, fruit and vegetables consumption and tobacco smoking, [36] while also decreasing heterogeneity $[2,47]$.

Studies comparing the results from published literature vs. individual participant data $[48,49]$ concluded that, although the direction and magnitude of the associations may differ between methods, the main difference and advantage of pooled analyses is the number of individuals/studies available, allowing for more precise and statistically significant estimates [49]. We have previously compared the same set of reports with the individual data present in the StoP Project database regarding the association between smoking and gastric cancer, and observed that, for all exposure categories considered, StoP included a larger number of studies and had more information available to perform stratified analyses, particularly regarding cancer location and amount of cigarettes smoked per day [50]. The same pattern was observed in the present work, namely regarding the number of drinks consumed per day, with the StoP database including a larger number of studies with this information and, therefore, allowing for a dose-response analysis, as previously shown [36]. In conclusion, the differences between the estimates obtained from published and individual participant data highlight the importance of individual participant pooled data for a more comprehensive and valid appraisal of the evidence available.

\section{Funding}

This study was funded by FEDER through the Operational Programme Competitiveness and Internationalization and national funding from the Foundation for Science and Technology - FCT (Portuguese Ministry of Science, Technology and Higher Education) under the Unidade de Investigação em Epidemiologia - Instituto de Saúde Pública da Universidade do Porto (EPIUnit) (POCI-01-0145FEDER-006862; Ref. UID/DTP/04750/2013); the PhD Grants PD/BD/ 105823/2014 (Ana Ferro) and SFRH/BD/102585/2014 (Samantha Morais) and the Postdoc grant SFRH/BPD/108751/2015 (Bárbara Peleteiro) co-funded by FCT and the "Programa Operacional Capital Humano" (POCH/FSE), the Fondazione Italiana per la Ricerca sul Cancro (FIRC), the Associazione Italiana per la Ricerca sul Cancro, project no. 16715 (Investigator Grant) and the Italian Ministry of Health (Young Researchers, GR-2011-02347943 to SB). Matteo Rota was supported by a fellowship from the FIRC.

The authors thank the European Cancer Prevention (ECP) Organization for providing support for the project meetings. We also thank all MCC-Spain study collaborators (CIBERESP, ISCIII, ISGlobal, ICO, University of Huelva, University of Oviedo, University of Cantabria, University of León, ibs. Granada, Instituto Salud Pública de Navarra, FISABIO, Murcia Regional Health Authority and cols).

\section{Conflict of interest}

There are no conflicts of interest to disclose.

\section{Authorship contribution statement}

The author contributions were as follows: AF collected, performed the statistical analysis and interpreted the data, drafted and revised the manuscript. SM collected and interpreted the data and revised the manuscript. MR, CP, PB, RB, CG harmonized the data, as part of the Stomach Cancer Pooling (StoP) Project. ZFZ, KM, HI, JH, KCJ, GPY, DP, MF, JM, RM, WY, HS, DZ, DM, NFL, MK, JV, EMNM, MP, FP, AW, NO, $\mathrm{AB}, \mathrm{NH}, \mathrm{LM}, \mathrm{RP}, \mathrm{RCK}, \mathrm{MHD}, \mathrm{AL}, \mathrm{PL}, \mathrm{PB}, \mathrm{SB}, \mathrm{EN}, \mathrm{BP}$ supplied the data, as part of the StoP Project. CLV and NL supervised the analysis and interpretation of data, and reviewed the manuscript. NL defined the study hypotheses and designed the investigation. All authors contributed to the discussion of the results. All authors read and approved the final version of the manuscript.

\section{Appendix A. Supplementary data}

Supplementary material related to this article can be found, in the online version, at doi:https://doi.org/10.1016/j.canep.2018.04.009.

\section{References}

[1] M. Egger, G.D. Smith, K. O'Rourke, Rationale, potentials and promise of systematic reviews, in: M. Egger, G.D. Smith, D. Altman (Eds.), Systemic Reviews in Health Care - meta-analysis in Context, BMJ Publishing Group, 2001, pp. 3-21.

[2] M. Blettner, W. Sauerbrei, B. Schlehofer, T. Scheuchenpflug, C. Friedenreich, Traditional reviews, meta-analyses and pooled analyses in epidemiology, Int. J. Epidemiol. 28 (1) (1999) 1-9.

[3] C.M. Friedenreich, Methods for pooled analyses of epidemiologic studies, Epidemiology 4 (4) (1993) 295-302.

[4] J.A. Sterne, D. Gavaghan, M. Egger, Publication and related bias in meta-analysis: power of statistical tests and prevalence in the literature, J. Clin. Epidemiol. 53 (11) (2000) 1119-1129.

[5] M. Simmonds, G. Stewart, L. Stewart, A decade of individual participant data metaanalyses: a review of current practice, Contemp. Clin. Trials 45 (Pt A) (2015) 76-83.

[6] World Cancer Research Fund International, American Institute for Cancer Research, Continuous Update Project Report: Diet, Nutrition, Physical Activity and Stomach Cancer, (2016)

[7] C. Pelucchi, N. Lunet, S. Boccia, Z.F. Zhang, D. Praud, P. Boffetta, F. Levi, K. Matsuo, H. Ito, J. Hu, K.C. Johnson, M. Ferraroni, G.P. Yu, B. Peleteiro, R. Malekzadeh, M.H. Derakhshan, W. Ye, D. Zaridze, D. Maximovitch, N. Aragones, V. Martin, M. Pakseresht, F. Pourfarzi, A. Bellavia, N. Orsini, A. Wolk, L. Mu, D. Arzani, R.C. Kurtz, P. Lagiou, D. Trichopoulos, J. Muscat, C. La Vecchia, E. Negri, The stomach cancer pooling (StoP) project: study design and presentation, Eur. J. Cancer Prev. 24 (1) (2015) 16-23.

[8] M. Rota, C. Pelucchi, P. Bertuccio, K. Matsuo, Z.F. Zhang, H. Ito, J. Hu, K.C. Johnson, D. Palli, M. Ferraroni, G.P. Yu, J. Muscat, N. Lunet, B. Peleteiro, W. Ye, H. Song, D. Zaridze, D. Maximovitch, M. Guevara, T. Fernandez-Villa, J. Vioque, E.M. Navarrete-Munoz, A. Wolk, N. Orsini, A. Bellavia, N. Hakansson, L. Mu, R. Persiani, R.C. Kurtz, A. Lagiou, P. Lagiou, C. Galeone, R. Bonzi, P. Boffetta, S. Boccia, E. Negri, C. La Vecchia, Alcohol consumption and gastric cancer risk-a pooled analysis within the StoP project consortium, Int. J. Cancer 141 (10) (2017) 1950-1962.

[9] I. Tramacere, E. Negri, C. Pelucchi, V. Bagnardi, M. Rota, L. Scotti, F. Islami, G. Corrao, C. La Vecchia, P. Boffetta, A meta-analysis on alcohol drinking and gastric cancer risk, Ann. Oncol. 23 (1) (2012) 28-36.

[10] I. Tramacere, C. Pelucchi, V. Bagnardi, M. Rota, L. Scotti, F. Islami, G. Corrao, P. Boffetta, C. La Vecchia, E. Negri, A meta-analysis on alcohol drinking and esophageal and gastric cardia adenocarcinoma risk, Ann. Oncol. 23 (2) (2012) 287-297.

[11] V. Bagnardi, M. Rota, E. Botteri, I. Tramacere, F. Islami, V. Fedirko, L. Scotti, M. Jenab, F. Turati, E. Pasquali, C. Pelucchi, C. Galeone, R. Bellocco, E. Negri, G. Corrao, P. Boffetta, C. La Vecchia, Alcohol consumption and site-specific cancer risk: a comprehensive dose-response meta-analysis, Br. J. Cancer 112 (3) (2015) 580-593.

[12] G.X. Tong, H. Liang, J. Chai, J. Cheng, R. Feng, P.L. Chen, Q.Q. Geng, X.R. Shen, D.B. Wang, Association of risk of gastric cancer and consumption of tobacco, alcohol and tea in the Chinese population, Asian Pac. J. Cancer Prev.: APJCP 15 (20) (2014) 8765-8774.

[13] H. Mahjub, G. Sadri, Association between alcohol consumption and gastric cancer: a meta-analysis, J. Res. Health Sci. 7 (2) (2007) 63-72.

[14] V. Bagnardi, M. Blangiardo, C. La Vecchia, G. Corrao, A meta-analysis of alcohol drinking and cancer risk, Br. J. Cancer 85 (11) (2001) 1700-1705.

[15] P. Lagiou, E. Samoli, A. Lagiou, J. Peterson, A. Tzonou, J. Dwyer, D. Trichopoulos, Flavonoids, vitamin c and adenocarcinoma of the stomach, Cancer Causes Control: CCC 15 (1) (2004) 67-72.

[16] E. Buiatti, D. Palli, A. Decarli, D. Amadori, C. Avellini, S. Bianchi, R. Biserni, F. Cipriani, P. Cocco, A. Giacosa, et al., A case-control study of gastric cancer and diet in Italy, Int. J. Cancer 44 (4) (1989) 611-616.

[17] E. De Feo, B. Simone, R. Persiani, F. Cananzi, A. Biondi, D. Arzani, R. Amore, D. D'Ugo, G. Ricciardi, S. Boccia, A case-control study on the effect of apolipo protein E genotypes on gastric cancer risk and progression, BMC Cancer 12 (2012) 494.

[18] C. La Vecchia, B. D’Avanzo, E. Negri, A. Decarli, J. Benichou, Attributable risks for 
stomach cancer in northern Italy, Int. J. Cancer 60 (6) (1995) 748-752.

[19] E. Lucenteforte, V. Scita, C. Bosetti, P. Bertuccio, E. Negri, C. La Vecchia, Food groups and alcoholic beverages and the risk of stomach cancer: a case-control study in Italy, Nutr. Cancer 60 (5) (2008) 577-584.

[20] N. Lunet, C. Valbuena, A.L. Vieira, C. Lopes, C. Lopes, L. David, F. Carneiro, $\mathrm{H}$. Barros, Fruit and vegetable consumption and gastric cancer by location and histological type: case-control and meta-analysis, Eur. J. Cancer Prev. 16 (4) (2007) 312-327.

[21] D. Zaridze, E. Borisova, D. Maximovitch, V. Chkhikvadze, Aspirin protects against gastric cancer: results of a case-control study from Moscow, Russia, Int. J. Cancer 82 (4) (1999) 473-476.

[22] G. Castano-Vinyals, N. Aragones, B. Perez-Gomez, V. Martin, J. Llorca, V. Moreno, J.M. Altzibar, E. Ardanaz, S. de Sanjose, J.J. Jimenez-Moleon, A. Tardon, J. Alguacil, R. Peiro, R. Marcos-Gragera, C. Navarro, M. Pollan, M. Kogevinas, M.C.S.S. Group, Population-based multicase-control study in common tumors in Spain (MCC-Spain): rationale and study design, Gac Sanit. 29 (4) (2015) 308-315.

[23] M. Santibanez, J. Alguacil, M.G. de la Hera, E.M. Navarrete-Munoz, J. Llorca, N. Aragones, T. Kauppinen, J. Vioque, P.S. Group, Occupational exposures and risk of stomach cancer by histological type, Occup. Environ. Med. 69 (4) (2012) 268-275.

[24] H. Harris, N. Håkansson, C. Olofsson, B. Julin, A. Åkesson, A. Wolk, The Swedish mammography cohort and the cohort of Swedish men: study design and characteristics of 2 population-based longitudinal cohorts, OA Epidemiol. 1 (2) (2013) 16.

[25] W. Ye, A.M. Ekstrom, L.E. Hansson, R. Bergstrom, O. Nyren, Tobacco, alcohol and the risk of gastric cancer by sub-site and histologic type, Int. J. Cancer 83 (2) (1999) 223-229.

[26] S. Deandrea, R. Foschi, C. Galeone, C. La Vecchia, E. Negri, J. Hu, Is temperature an effect modifier of the association between green tea intake and gastric cancer risk? Eur J. Cancer Prev. 19 (1) (2010) 18-22.

[27] L.N. Mu, Q.Y. Lu, S.Z. Yu, Q.W. Jiang, W. Cao, N.C. You, V.W. Setiawan, X.F. Zhou, B.G. Ding, R.H. Wang, J. Zhao, L. Cai, J.Y. Rao, D. Heber, Z.F. Zhang, Green tea drinking and multigenetic index on the risk of stomach cancer in a Chinese population, Int. J. Cancer 116 (6) (2005) 972-983.

[28] V.W. Setiawan, G.P. Yu, Q.Y. Lu, M.L. Lu, S.Z. Yu, L. Mu, J.G. Zhang, R.C. Kurtz, L. Cai, C.C. Hsieh, Z.F. Zhang, Allium vegetables and stomach cancer risk in China, Asian Pac. J. Cancer Prev.: APJCP 6 (3) (2005) 387-395.

[29] V.W. Setiawan, Z.F. Zhang, G.P. Yu, Y.L. Li, M.L. Lu, C.J. Tsai, D. Cordova, M.R. Wang, C.H. Guo, S.Z. Yu, R.C. Kurtz, GSTT1 and GSTM1 null genotypes and the risk of gastric cancer: a case-control study in a Chinese population, Cancer Epidemiol. Biomark. Prev. : Publ. Am. Assoc. Cancer Res. Cosponsored Am. Soc. Prevent. Oncol. 9 (1) (2000) 73-80.

[30] M.H. Derakhshan, R. Malekzadeh, H. Watabe, A. Yazdanbod, V. Fyfe, A. Kazemi, N. Rakhshani, R. Didevar, M. Sotoudeh, A.A. Zolfeghari, K.E. McColl, Combination of gastric atrophy, reflux symptoms and histological subtype indicates two distinct aetiologies of gastric cardia cancer, Gut 57 (3) (2008) 298-305.

[31] M. Pakseresht, D. Forman, R. Malekzadeh, A. Yazdanbod, R.M. West, D.C. Greenwood, J.E. Crabtree, J.E. Cade, Dietary habits and gastric cancer risk in north-west Iran, Cancer Causes Control: CCC 22 (5) (2011) 725-736.

[32] F. Pourfarzi, A. Whelan, J. Kaldor, R. Malekzadeh, The role of diet and other environmental factors in the causation of gastric cancer in Iran-a population based study, Int. J. Cancer 125 (8) (2009) 1953-1960.

[33] K. Matsuo, I. Oze, S. Hosono, H. Ito, M. Watanabe, K. Ishioka, S. Ito, M. Tajika, Y. Yatabe, Y. Niwa, K. Yamao, S. Nakamura, K. Tajima, H. Tanaka, The aldehyde dehydrogenase 2 (ALDH2) Glu504Lys polymorphism interacts with alcohol drinking in the risk of stomach cancer, Carcinogenesis 34 (7) (2013) 1510-1515.

[34] Y. Mao, J. Hu, R. Semenciw, K. White, G. Canadian, Cancer registries epidemiology research, active and passive smoking and the risk of stomach cancer, by subsite, in
Canada, Eur. J. Cancer Prev. 11 (1) (2002) 27-38.

[35] Z.F Zhang R.C. Kurtz, D. S. Klimstra, G.P. Yu, M. Sun, S. Harlap, J. R. Marshall, Helicobacter pylori infection on the risk of stomach cancer and chronic atrophic gastritis, Cancer Detect Prev. 23 (5) (1999) 357-367.

[36] R. DerSimonian, N. Laird, Meta-analysis in clinical trials revisited, Contemp. Clin. Trials 45 (Pt A) (2015) 139-145.

[37] J.P. Higgins, S.G. Thompson, Quantifying heterogeneity in a meta-analysis, Stat. Med. 21 (11) (2002) 1539-1558.

[38] C. Turner, How much alcohol is in a' standard drink'? An analysis of 125 studies, Br. J. Addict. 85 (9) (1990) 1171-1175.

[39] S.J. Nevitt, A.G. Marson, B. Davie, S. Reynolds, L. Williams, C.T. Smith, Exploring changes over time and characteristics associated with data retrieval across individual participant data meta-analyses: systematic review, BMJ (Clin. Res. Ed.) 357 (2017) j1390.

[40] J.R. Polanin, Efforts to retrieve individual participant data sets for use in a metaanalysis result in moderate data sharing but many data sets remain missing, J. Clin. Epidemiol. (2017).

[41] J.F. Hu, S.F. Zhang, E.M. Jia, Q.Q. Wang, S.D. Liu, Y.Y. Liu, Y.P. Wu, Y.T. Cheng, Diet and cancer of the stomach: a case-control study in China, Int. J. Cancer 41 (3) (1988) 331-335.

[42] C.M. Gao, T. Takezaki, J.H. Ding, M.S. Li, K. Tajima, Protective effect of allium vegetables against both esophageal and stomach cancer: a simultaneous case-referent study of a high-epidemic area in jiangsu Province, China, Jpn. J. Cancer Res. Gann 90 (6) (1999) 614-621.

[43] G.P. Yu, C.C. Hsieh, L.Y. Wang, S.Z. Yu, X.L. Li, T.H. Jin, Green-tea consumption and risk of stomach cancer: a population-based case-control study in Shanghai, China, Cancer Causes Control: CCC 6 (6) (1995) 532-538.

[44] S.L. Park, D. Bastani, B.Y. Goldstein, S.C. Chang, W. Cozen, L. Cai, C. Cordon-Cardo, B. Ding, S. Greenland, N. He, S.K. Hussain, Q. Jiang, Y.C. Lee, S. Liu, M.L. Lu, T.M. Mack, J.T. Mao, H. Morgenstern, L.N. Mu, S.S. Oh, A. Pantuck, J.C. Papp, J. Rao, V.E. Reuter, D.P. Tashkin, H. Wang, N.C. You, S.Z. Yu, J.K. Zhao, Z.F. Zhang, Associations between NBS1 polymorphisms, haplotypes and smoking-related cancers, Carcinogenesis 31 (7) (2010) 1264-1271.

[45] J. Key, S. Hodgson, R.Z. Omar, T.K. Jensen, S.G. Thompson, A.R. Boobis, D.S. Davies, P. Elliott, Meta-analysis of studies of alcohol and breast cancer with consideration of the methodological issues, Cancer Causes Control: CCC 17 (6) (2006) 759-770.

[46] C. Zeisser, T.R. Stockwell, T. Chikritzhs, Methodological biases in estimating the relationship between alcohol consumption and breast cancer: the role of drinker misclassification errors in meta-analytic results, Alcohol. Clin. Exp. Res. 38 (8) (2014) 2297-2306.

[47] M. Egger, M. Schneider, G. Davey Smith, Spurious precision? Meta-analysis of observational studies, BMJ (Clin. Res. Ed.) 316 (7125) (1998) 140-144.

[48] L. Stewart, M. Parmar, Meta-analysis of the literature or of individual patient data: is there a difference? Lancet 341 (8842) (1993) 418-422.

[49] C. Tudur Smith, M. Marcucci, S.J. Nolan, A. Iorio, M. Sudell, R. Riley, M.M. Rovers, P.R. Williamson, Individual participant data meta-analyses compared with metaanalyses based on aggregate data, Cochr. Datab. Syst. Rev. (2016) Mr000007.

[50] A. Ferro, S. Morais, M. Rota, C. Pelucchi, P. Bertuccio, R. Bonzi, C. Galeone, Z.F. Zhang, K. Matsuo, H. Ito, J. Hu, K.C. Johnson, G.P. Yu, D. Palli, M. Ferraroni, J. Muscat, R. Malekzadeh, W. Ye, H. Song, D. Zaridze, D. Maximovitch, N. Aragones, G. Castano-Vinyals, J. Vioque, E.M. Navarrete-Munoz, M. Pakseresht, F. Pourfarzi, A. Wolk, N. Orsini, A. Bellavia, N. Hakansson, L. Mu, R. Pastorino, R.C. Kurtz, M.H. Derakhshan, A. Lagiou, P. Lagiou, P. Boffetta, S. Boccia, E. Negri, C. La Vecchia, B. Peleteiro, N. Lunet, Tobacco smoking and gastric cancer: metaanalyses of published data versus pooled analyses of individual participant data (StoP Project), Eur J. Cancer Prev. 27 (3) (2018) 197-204. 